\title{
BioéthiqueOnline
}

\section{Exploration des dilemmes éthiques entourant le traitement de la dysphagie à l'enfance et leurs solutions : perceptions d'intervenants}

\author{
Anne-Marie Brûlé et Marie-Josée Drolet
}

Volume 6, 2017

URI : https://id.erudit.org/iderudit/1044617ar

DOI : https://doi.org/10.7202/1044617ar

Aller au sommaire du numéro

Éditeur(s)

BioéthiqueOnline

ISSN

1923-2799 (numérique)

Découvrir la revue

Citer cet article

Brûlé, A.-M. \& Drolet, M.-J. (2017). Exploration des dilemmes éthiques entourant le traitement de la dysphagie à l'enfance et leurs solutions : perceptions d'intervenants. BioéthiqueOnline, 6 .

https://doi.org/10.7202/1044617ar
Résumé de l'article

Une recension des écrits révèle qu'il existe peu de connaissances sur les dilemmes éthiques (et leurs solutions) entourant le traitement de la dysphagie à l'enfance. Le but de cette étude était d'explorer, voire de décrire les dilemmes éthiques rencontrés par des intervenants lors du traitement des problèmes d'alimentation chez des enfants souffrant de dysphagie et les façons dont ceux-ci procèdent pour les résoudre. Des entrevues semi-dirigées ont été réalisées auprès de huit intervenantes travaillant dans un Centre intégré universitaire de santé et de services sociaux (CIUSSS) du Québec. Toutes les intervenantes mentionnent avoir rencontré des dilemmes éthiques, et ce, en moyenne deux fois par année et ceux-ci leur ont fait vivre une certaine détresse. Ces dilemmes ont trois causes principales, soit les refus de traitement de certains parents, les suivis partiels des recommandations professionnelles et les divergences d'opinions avec des partenaires externes. Pour résoudre ces dilemmes, les intervenantes affirment recourir à des discussions en équipe et à de l'aide de supérieurs, de partenaires externes ou de parents. La majorité des intervenantes mentionnent avoir besoin de moyens supplémentaires pour résoudre ces dilemmes et proposent certaines avenues en ce sens. Les résultats de la recherche rejoignent en général ceux documentés dans les écrits. Plus d'attention devrait être portée à ces dilemmes étant donné le peu de ressources éthiques, actuellement disponibles, adaptées à ces situations pour les résoudre et la détresse que ceux-ci occasionnent.
Droits d'auteur (c) Anne-Marie Brûlé et Marie-Josée Drolet, 2017

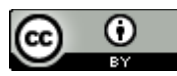

Ce document est protégé par la loi sur le droit d'auteur. L’utilisation des services d’Érudit (y compris la reproduction) est assujettie à sa politique d'utilisation que vous pouvez consulter en ligne. 


\title{
Exploration des dilemmes éthiques entourant le traitement de la dysphagie à l'enfance et leurs solutions : perceptions d'intervenants
}

ARTICLE (RÉVISION PAR LES PAIRS / PEER-REVIEWED)

Anne-Marie Brûlé ${ }^{1}$, Marie-Josée Drolet ${ }^{2}$

\author{
Reçu/Received: 21 Aug 2015 \\ Publié/Published: 26 Sept 2017 \\ Éditeurs/Editors: Charles Dupras, Vanessa Chenel, Aliya Affdal, Mariana Nunez \\ Évaluateurs externes/Peer-Reviewers: Vincent Martel-Sauvageau \& Audette Sylvestre
}

2017 A-M Brûlé, M-J Drolet, Creative Commons Attribution 4.0 International License

\section{Résumé}

Une recension des écrits révèle qu'il existe peu de connaissances sur les dilemmes éthiques (et leurs solutions) entourant le traitement de la dysphagie à l'enfance. Le but de cette étude était d'explorer, voire de décrire les dilemmes éthiques rencontrés par des intervenants lors du traitement des problèmes d'alimentation chez des enfants souffrant de dysphagie et les façons dont ceux-ci procèdent pour les résoudre. Des entrevues semi-dirigées ont été réalisées auprès de huit intervenantes travaillant dans un Centre intégré universitaire de santé et de services sociaux (CIUSSS) du Québec. Toutes les intervenantes mentionnent avoir rencontré des dilemmes éthiques, et ce, en moyenne deux fois par année et ceux-ci leur ont fait vivre une certaine détresse. Ces dilemmes ont trois causes principales, soit les refus de traitement de certains parents, les suivis partiels des recommandations professionnelles et les divergences d'opinions avec des partenaires externes. Pour résoudre ces dilemmes, les intervenantes affirment recourir à des discussions en équipe et à de l'aide de supérieurs, de partenaires externes ou de parents. La majorité des intervenantes mentionnent avoir besoin de moyens supplémentaires pour résoudre ces dilemmes et proposent certaines avenues en ce sens. Les résultats de la recherche rejoignent en général ceux documentés dans les écrits. Plus d'attention devrait être portée à ces dilemmes étant donné le peu de ressources éthiques, actuellement disponibles, adaptées à ces situations pour les résoudre et la détresse que ceux-ci occasionnent.

\section{Mots clés}

éthique, dilemme, détresse, dysphagie, enfance, perception, intervenant, phénoménologie

\section{Abstract}

A review of the literature reveals that there is little knowledge about the ethical dilemmas (and their solutions) surrounding the treatment of childhood dysphagia. The aim of this study is to explore and describe the ethical dilemmas faced by practitioners in the treatment of feeding problems in children with dysphagia and the ways in which these problems are solved. Semi-directed interviews were conducted with eight health practitioners working in an integrated university health and social services centre (CIUSSS) in Quebec. All the practitioners mentioned that they had encountered ethical dilemmas, on average twice a year, and these had caused them some distress. These dilemmas have three main causes: refusal of treatment by some parents, partial follow-up of professional recommendations and differences of opinion with external partners. To resolve these dilemmas, practitioners say they rely on team discussions and the help of superiors, external partners or parents. The majority of participants mentioned that they needed additional means to resolve these dilemmas and proposed some avenues in that direction. The results of the research generally correspond to those documented in the literature. More attention should be paid to these dilemmas, given the scarcity of ethical resources currently available, that are adapted to and able to address these situations, and the distress that these cause.

\section{Keywords}

ethics, dilemma, distress, dysphagia, childhood, perception, intervener, phenomenology

\section{Responsabilités des évaluateurs externes}

Les évaluations des examinateurs externes sont prises en considération de façon sérieuse par les éditeurs et les auteurs dans la préparation des manuscrits pour publication. Toutefois, être nommé comme examinateur n'indique pas nécessairement l'approbation de ce manuscrit. Les éditeurs de BioéthiqueOnline assument la responsabilité entière de l'acceptation finale et la publication d'un article.
Peer-reviewer responsibilities

Reviewer evaluations are given serious consideration by the editors and authors in the preparation of manuscripts for publication. Nonetheless, being named as a reviewer does not necessarily denote approval of a manuscript; the editors of BioéthiqueOnline take full responsibility for final acceptance and publication of an article. 
Affiliations des auteurs / Author Affiliations

${ }^{1}$ Centre intégré de santé et de services sociaux de Lanaudière, Joliette, Canada

${ }^{2}$ Département d'ergothérapie, Université du Québec à Trois-Rivières, Trois-Rivières, Canada

\section{Correspondance / Correspondence}

Anne-Marie Brûlé, ambrule25@hotmail.com

\section{Remerciements}

Les auteures remercient les participantes à la recherche, sans lesquelles celle-ci n'aurait pas pu être réalisée. Elles témoignent également leur reconnaissance aux personnes du CIUSSS participant qui les ont aidées tout au long de la recherche. Elles remercient également les éditeurs et réviseurs de la revue qui leur ont permis de clarifier leurs idées et de bonifier de manière générale l'article.

\section{Conflit d'intérêts}

Les auteurs ne rapportent aucun conflit d'intérêts reliés à la conduite de cette recherche ni source de financement. Mentionnons cependant qu'Anne-Marie Brûlé a obtenu trois bourses d'études, à savoir la bourse pour avoir eu un cheminement particulier n'ayant pas favorisé la poursuite des études (maternité), la bourse d'excellence dédiée à l'étudiant ayant obtenu la meilleure moyenne au baccalauréat en ergothérapie et la bourse d'excellence pour l'admission à la maîtrise en ergothérapie. Par ailleurs, Marie-Josée Drolet est détentrice de plusieurs subventions de recherche afin de documenter les enjeux éthiques de la pratique ergothérapique. Les organismes subventionnaires suivants soutiennent ses projets: CRSH, FRQSC, Fondation de I'UQTR et UQTR. Mentionnons cependant que ces fonds n'ont pas financé la présente étude qui s'inscrivait dans le cadre de la maîtrise en ergothérapie d'Anne-Marie Brûlé.

\section{Acknowledgements}

The authors thank the research participants, without whom this research could not have been carried out. They also express their gratitude to the participants of the CIUSSS who helped them throughout the research. They also thank the journal editors and peer-reviewers who helped them to clarify their ideas and improve the article in general.

\section{Conflicts of Interest}

The authors do not have any conflicts of interest related to the conduct of this study nor its source of funding. It should be noted, however, that Anne-Marie Brûlé received three scholarships, namely the scholarship for having had a particular path that did not favour the continuation of studies (maternity), an excellence scholarship for the student who obtained the best average at the bachelor's degree in occupational therapy, and the excellence scholarship for admission to the master's program in occupational therapy. In addition, Marie-Josée Drolet holds several research grants to document ethical issues in occupational therapy practice. The following granting agencies support her projects: CRSH, FRQSC, UQTR Foundation and UQTR. It should be noted, however, that these funds did not fund this study, which was part of the Anne-Marie Brûlé's master's in occupational therapy.

\section{Introduction}

L'activité de s'alimenter ou les préoccupations entourant l'acte de s'alimenter font partie intégrante du quotidien des êtres humains. Dès les tout premiers jours de la vie, l'alimentation constitue une activité quotidienne à la fois nécessaire et significative pour tout individu, en outre parce que celle-ci est essentielle au bien-être et à la vie des personnes [1]. Or, les problèmes d'alimentation sont assez fréquents, notamment chez les enfants. En effet, selon Arvedson et ses collaborateurs [2], la prévalence des problèmes d'alimentation chez les enfants ayant un développement typique varie entre $25 \%$ et $40 \%$ ainsi qu'entre $33 \%$ et $80 \%$ chez ceux présentant un retard de développement. Ces problèmes ont soit une cause comportementale ou physiologique. Aussi, parmi les problèmes d'alimentation ayant une cause physiologique se trouve la dysphagie [2].

La dysphagie correspond à la difficulté rencontrée par une personne à transférer la nourriture de la cavité orale vers l'estomac, en passant par le pharynx et l'œsophage [3]. Elle peut être permanente ou transitoire, intermittente ou progressive ainsi que d'intensité variable selon les individus. De fait, la dysphagie se manifeste de diverses manières, allant du simple inconfort jusqu'à des difficultés majeures pouvant mettre en péril la vie des personnes atteintes. Les conséquences de la dysphagie sont nombreuses et importantes pour ces dernières, notamment pour l'enfant présentant une dysphagie, ses parents ou tuteurs, les intervenants impliqués, le cas échéant, et même pour la société en général [3]. Elle peut par exemple avoir des conséquences d'ordre physique comme une infection des voies respiratoires, la dénutrition et, parfois même, la mort de l'individu qui en souffre [4]. Elle peut aussi par moments avoir des conséquences d'ordre psychologique, culturel ou social. Par exemple, elle peut être une source d'anxiété pour la personne atteinte et ses proches ainsi que favoriser l'isolement social de celle-ci et de ses proches [3,5]. 
Au Québec, dépendamment des établissements de santé, différents intervenants peuvent être impliqués dans le traitement de la dysphagie à l'enfance comme l'ergothérapeute, l'infirmier, le médecin, le nutritionniste et l'orthophoniste. Les champs de pratique de ces intervenants étant différents, mais complémentaires, il en est de même pour leurs rôles et responsabilités dans le traitement de la dysphagie. Par exemple, tandis que le médecin établit le diagnostic de dysphagie en collaboration avec l'infirmier, le nutritionniste élabore des menus propres à la dysphagie visant à contrer la dénutrition en s'assurant en outre que la texture des aliments soit propice à la déglutition. L'ergothérapeute assure un positionnement sécurisé de la personne atteinte et propose des aides techniques ainsi que des exercices qui soutiennent son autonomie, tandis que l'orthophoniste, qui occupe un rôle crucial dans l'évaluation et le traitement de la dysphagie, porte une attention particulière aux régions du tractus aéro-digestif supérieur de la personne atteinte pour contrer à la source les conséquences négatives de la dysphagie [6]. II arrive que l'inhalothérapeute, le kinésiologue, le psychologue ou le travailleur social collaborent avec ces professionnels dans le traitement de la dysphagie, mais leur participation est plus rare et déterminée selon leur expertise propre en fonction des besoins de la personne, en l'occurrence l'enfant et sa famille.

Le traitement de la dysphagie à l'enfance peut engendrer des problèmes d'ordre éthique à la fois chez les intervenants et les familles, et ce, surtout lorsque les interventions se révèlent inefficaces [7]. II arrive, en effet, que les traitements prodigués ne soient pas efficaces et que l'alimentation orale soit non sécuritaire, en raison notamment d'un haut taux d'aspiration (c'est-à-dire l'entrée de corps étrangers dans les voies respiratoires sous les cordes vocales) qui peut avoir des conséquences négatives non seulement sur le statut nutritionnel de l'enfant (risque de dénutrition), mais également sur sa santé respiratoire (risque d'infections pulmonaires qui peuvent mener au décès de l'enfant). De fait, lorsque l'alimentation orale met en péril le statut nutritionnel, la santé pulmonaire ou même la vie de l'enfant [8], les familles et les intervenants peuvent être confrontés à des dilemmes éthiques importants et difficiles à gérer [7].

Un dilemme éthique correspond à une situation problématique sur le plan de l'éthique au sein de laquelle une personne est confrontée à un choix déchirant [9]. Dans ce genre de situation, bien que les options s'offrant à l'individu soient désirables, celles-ci étant inconciliables (ou difficilement conciliables), l'individu vit de l'incertitude quant à l'option devant être privilégiée. Comme le résument Swisher et ses collaborateurs [9], ce genre de situation oppose au moins deux possibilités d'action qui reposent au final sur des valeurs éthiques irréconciliables (ou difficiles à concilier) et parmi lesquelles un choix doit généralement être fait. Par exemple, dans les cas de dysphagie à l'enfance, un intervenant ou un parent pourrait être partagé entre la cessation de l'alimentation orale ou l'installation d'un tube nasogastrique. Mentionnons qu'un tube nasogastrique permet d'accéder à la cavité gastrique par la voie nasale et il a pour fonction notamment: 1) d'effectuer un lavage gastrique; 2) d'aspirer des sécrétions gastriques; 3) d'effectuer un tubage gastrique; 4) d'administrer un traitement; et 5) de permettre une alimentation entérale [12]. Advenant une telle situation, faut-il poursuivre l'alimentation orale de l'enfant en modifiant les textures des aliments, mais en risquant ce faisant les infections pulmonaires? Devrait-on plutôt poursuivre l'alimentation orale de l'enfant au risque de sa mort par étouffement ou installer un tube gastrique assurant ainsi sa sécurité alimentaire, mais affectant ce faisant à la négative sa qualité de vie? Dans ces cas, les intervenants et les parents peuvent être confrontés à des dilemmes éthiques, lesquels peuvent être complexifiés par le fait que les intervenants et les parents ont par moments des points de vue différents au sujet du meilleur intérêt de l'enfant [7]. Par exemple, bien que la plupart des parents consentent à l'alimentation par tube nasogastrique, certains refusent cette intervention pour diverses raisons comme la qualité de vie de leur enfant par exemple [4], ce qui peut être vécu difficilement par certains intervenants qui ont de réelles craintes pour la sécurité ou la vie de l'enfant.

II s'ensuit que le fait de vivre des dilemmes éthiques peut avoir des conséquences négatives à la fois chez les parents et les intervenants [7,13]. Chez les parents, cela est susceptible d'occasionner des sentiments comme l'impuissance, l'ambivalence, l'échec, la perte d'espoir ainsi que l'abandon de 
l'idée de la soi-disant normalité de leur enfant [14]. Chez les intervenants, cela peut avoir des conséquences négatives comme l'épuisement professionnel [15], le changement de carrière [15,16], la recherche d'un emploi dans un autre établissement, la diminution du nombre d'heures travaillées, la condamnation de l'établissement ou de ses administrateurs, l'évitement des interactions avec l'enfant et sa famille, la diminution de la qualité des relations interprofessionnelles [17], et ce, au point de compromettre leur intégrité morale $[18,19]$. De plus, cela est susceptible d'engendrer de la détresse éthique chez certains intervenants [15,20], ce qui peut occasionner une certaine détresse psychologique [9]. Mentionnons que la détresse éthique correspond à une situation où l'intervenant ne parvient pas à actualiser une ou des valeurs éthiques importantes pour lui en raison de barrières externes à sa personne, souvent de nature institutionnelle [9]. Bien que cette détresse puisse avoir des conséquences positives, notamment amener les intervenants à être plus conscients de leurs valeurs [21], améliorer leur habileté à résoudre des problèmes éthiques, initier un processus d'apprentissage et améliorer la qualité des services prodigués [19], les conséquences négatives sur les intervenants ne saurait être négligées puisqu'elles contribuent à les épuiser affectivement et peuvent avoir des conséquences sociales négatives non négligeables [15,20,22]. Somme toute, les dilemmes éthiques, dont ceux que suscite le traitement de la dysphagie à l'enfance, occasionnent des conséquences négatives chez les parents et les intervenants, bien que certaines conséquences positives comme celles nommées ci-haut puissent aussi en résulter pour ces derniers.

À ce jour, les dilemmes éthiques entourant le traitement de la dysphagie à l'enfance sont peu documentés dans les écrits et il en est de même des solutions pouvant être mises de l'avant afin de les résoudre [23]. Compte tenu des difficultés rencontrées par les intervenants et les familles à surmonter ce genre de situations qui engendre de nombreuses conséquences négatives, il importe de les documenter, de même que les manières de les résoudre afin d'identifier des solutions pouvant être pertinentes et actualisables en pratique. Cette recherche est également justifiée d'un point de vue professionnel parce qu'elle entend offrir des pistes de solution pour soutenir les intervenants dans leurs prises de décisions. Elle est aussi pertinente d'un point de vue social parce qu'elle vise, certes modestement et indirectement, à favoriser la santé, la sécurité, la qualité de vie et la vie des enfants présentant un problème de dysphagie. En outillant les intervenants et les familles à surmonter les dilemmes éthiques que suscitent ces situations, il est souhaité qu'ils soient habiletés à mieux les résoudre. II s'ensuit que l'objectif de cette recherche était d'explorer, voire de décrire, les perceptions d'intervenants des dilemmes éthiques qu'ils rencontrent lors du traitement des problèmes d'alimentation d'enfants souffrant de dysphagie, de même que des manières dont ils les solutionnent, le cas échéant. Cette recherche s'avère donc justifiée d'un point de vue scientifique parce qu'elle contribue à l'édification des connaissances dans un domaine encore peu exploré par les chercheurs.

\section{Méthode}

\section{Devis de la recherche}

Comme l'état actuel des connaissances relatif aux dilemmes éthiques entourant le traitement des enfants ayant un problème dysphagique et aux solutions pouvant être mises de l'avant est limité, un devis qualitatif était indiqué pour atteindre l'objectif de l'étude [23]. Aussi, puisque l'objet interrogé consiste en des perceptions d'intervenants, un devis d'inspiration phénoménologique fut utilisé, lequel puise à même la phénoménologie descriptive et transcendantale du philosophe Husserl [24]. La phénoménologie husserlienne est une philosophie herméneutique dont la finalité consiste à accéder à l'essence des phénomènes par l'entremise des perceptions humaines. Ce devis était dès lors approprié, car il permet de décrire les perceptions des personnes directement confrontées aux phénomènes interrogés dans le but d'en faire émerger l'essence, et ce, du point de vue des personnes les ayant directement vécus $[23,25,26]$. 


\section{Participants à l'étude}

Comme la recherche répondait à une demande du milieu (des intervenants confrontés à de tels dilemmes avaient approché la deuxième auteure de l'article afin d'être mieux outillés pour aborder ce genre de situations) et que les situations problématiques devaient être d'abord clarifiées pour ensuite trouver des solutions appropriées, il fut décidé qu'une première étape serait de décrire les dilemmes et les solutions mises en œuvre dans le milieu. Un agent de planification de programme et de recherche du Centre intégré universitaire de santé et de services sociaux (CIUSSS), responsable du recrutement, s'est assuré que les intervenants retenus rencontraient les critères d'inclusion de l'étude, soit : 1) être un professionnel enregistré à l'ordre professionnel le concernant; 2) œuvrer au sein du CIUSSS participant à la recherche; 3) travailler ou avoir travaillé au sein d'une équipe pluridisciplinaire ayant pour mandat de traiter des enfants ayant un problème de dysphagie; et 4) vivre présentement ou avoir vécu des dilemmes éthiques en lien avec cette pratique. La notion de dilemme éthique avait été définie dans la lettre de recrutement des participants qui a été remise à l'agent responsable du recrutement. Enfin, les intervenants devaient également parler et comprendre le français ainsi qu'être volontaires et disponibles pour participer à la recherche. Aucun critère d'exclusion n'a été utilisé lors du recrutement des participants. Enfin, par l'entremise de la méthode d'échantillonnage par choix raisonné $[23,25]$, six à dix intervenants étaient souhaités afin d'atteindre la saturation des données [27].

\section{Collecte et analyse des données}

Les données de la recherche ont été collectées par l'entremise d'entrevues semi-dirigées individuelles d'une durée variant entre 60 et 90 minutes selon les participants. Les entrevues avec les intervenants ont été réalisées sur leur lieu de travail et enregistrées sur bande audionumérique pour permettre la transcription des verbatim. Le schéma de l'entrevue comprenait trois sections : une première recueillant des données descriptives sur les participants, une seconde abordant les dilemmes éthiques et une dernière dédiée aux solutions mises de l'avant par les participants pour les résoudre. Suivant les principes du devis phénoménologique, les entrevues étaient essentiellement constituées de questions larges, ouvertes et non directives [23-26]. Par exemple, "Décrivez des situations où vous avez fait l'expérience de dilemmes éthiques en lien avec le traitement d'enfants ayant une dysphagie? " ou encore "De quelle manière ces dilemmes ont-ils été résolus, le cas échéant?».

Une analyse qualitative des verbatim a été réalisée par l'auteure principale, soit une analyse de contenu, laquelle consiste à organiser et à interpréter les propos des participants pour en découvrir le sens $[23,25,26]$. Plus précisément, les étapes proposées par Giorgi afin d'appliquer la phénoménologie de Husserl ont été réalisées [28], à savoir : la lecture des données, la création des unités de sens, l'organisation et la formulation des données dans le langage disciplinaire et la synthèse des résultats $[26,28]$. Enfin, la majorité des participants à l'étude ont lu et commenté les analyses des verbatim afin d'assurer leurs conformités à leurs propos, perceptions et idées. Cette façon de fonctionner visait à valider les interprétations des données et à assurer la crédibilité des résultats.

\section{Considérations éthiques}

Deux certifications éthiques ont été obtenues pour mener à terme cette recherche : une du Comité d'éthique de la recherche avec des êtres humains de l'Université du Québec à Trois-Rivières et une autre du Comité d'éthique de la recherche du CIUSSS concerné. Un formulaire de consentement écrit a été signé par tous les participants à la recherche qui étaient libres d'y prend part ou non. Enfin, la confidentialité des participants a été assurée en leur assignant un numéro et en évitant de donner des informations précises à leur sujet lors de la diffusion des résultats. 


\section{Résultats}

\section{Description des participants}

Huit intervenants ont participé à la recherche, tous des femmes ${ }^{1}$. Au moment de la collecte des données, celles-ci étaient âgées entre 31 et 53 ans. Leur âge moyen était de 41 ans avec un écart type de 6,7 ans. Au sujet du nombre d'années de pratique professionnelle, les participantes avaient entre 8 et 31 années d'expérience, pour une moyenne de 18 ans avec un écart type de 6,9 ans. Parmi les participantes, deux étaient coordonnatrices cliniques (ergothérapeutes), deux ergothérapeutes cliniciennes, deux orthophonistes, une nutritionniste et une travailleuse sociale. Enfin, deux participantes avaient suivi par le passé une formation à l'éthique d'au moins une journée.

\section{Perceptions des intervenantes des dilemmes éthiques}

Toutes les intervenantes rencontrées en entrevue affirment vivre des situations de dilemmes éthiques entourant le traitement d'enfants ayant des problèmes dysphagiques. La fréquence moyenne estimée par les intervenantes est d'au moins deux dilemmes éthiques par année. Bien que ces situations soient peu fréquentes, lorsque vécues les intervenantes mentionnent vivre une certaine détresse. Selon elles, ces situations de grands doutes quant à la décision et à l'action à réaliser ont trois sources principales : 1) les refus de traitement de certains parents, 2) les suivis partiels de certains parents des recommandations professionnelles ou 3) les divergences d'opinions avec des partenaires externes.

\section{Refus de traitement de certains parents}

Les situations de refus de traitement de certains parents sont source de dilemmes éthiques chez plusieurs des intervenantes rencontrées. Bien qu'elles valorisent le respect et le soutien de l'autonomie décisionnelle des parents, il leur est parfois difficile d'accepter sereinement les refus de traitement, notamment lorsque ces refus, estiment-elles, sont susceptibles de compromettre la santé, la sécurité ou encore la vie de l'enfant atteint de dysphagie. Ayant à cœur la protection des personnes vulnérables que sont ces enfants, elles ont parfois le sentiment que certains parents qui usent de leur droit de refuser certaines interventions ne comprennent peut-être pas suffisamment les risques, et ce, malgré que toutes les informations pertinentes leur aient été, selon leur avis, transmises. Ce faisant, elles sont partagées quant à la façon d'intervenir.

- Comment assurer un consentement aux soins qui soit véritablement libre, c'est-à-dire sans pression indue de la part de l'équipe?

- Comment assurer un consentement qui soit suffisamment éclairé par toute l'information pertinente et adaptée aux capacités de compréhension des parents?

- Comment assurer la santé, la sécurité et la vie des enfants, tout en laissant les parents libres de déterminer ce qui convient de faire pour répondre aux meilleurs intérêts de leur enfant?

- Comment, tout en voulant protéger les personnes vulnérables que sont les enfants, ne pas user de paternalisme médical ou éthique à l'endroit des parents qui semblent, selon l'équipe, prendre des risques alimentaires injustifiés, car trop risqués?

Les paragraphes qui suivent donnent quatre exemples de situations cliniques ayant engendré ce genre de dilemmes éthiques.

Par exemple, une intervenante rapporte une situation qui concerne une fillette de quatre ans et demi dont les parents ont refusé l'installation d'un gavage, alors que celle-ci en aurait bénéficié, selon elle, en raison de son hydratation et son statut pondéral insuffisants. Dans cette situation, l'intervenante estimait que la santé nutritionnelle de l'enfant était en conflit avec l'autonomie décisionnelle des parents.

\footnotetext{
${ }^{1}$ À partir d'ici, le féminin est utilisé étant donné que tous les participants à l'étude étaient des femmes.
} 
Une intervenante discute d'un garçon d'environ seize ans qui avait une maladie dégénérative. L'équipe recommandait aux parents la gastrostomie - qui correspond à un dispositif donnant un accès direct à l'estomac pour réaliser une nutrition entérale [30] -, mais ceux-ci n'étaient pas prêts à accepter cette opération et refusaient pour le motif que manger était le seul plaisir qui restait à leur fils. Dans cette situation, la santé et la sécurité alimentaires du garçon s'opposaient à la qualité de vie de l'enfant et à l'autonomie décisionnelle des parents.

Des intervenantes rapportent une situation vécue par une adolescente âgée de 18 ans. Celle-ci était nourrie exclusivement par voie orale au moyen de purées lisses, mais des tests ont révélé qu'elle s'aspirait beaucoup. II avait alors été recommandé à la mère de procéder à l'installation d'un tube nasogastrique. Les parents avaient refusé et décidé de continuer l'alimentation par voie orale. Les raisons évoquées par ceux-ci référaient au plaisir et à la qualité de vie de leur fille. Encore ici, la santé et la sécurité alimentaires de l'adolescente étaient en tension avec la qualité de vie de celle-ci et l'autonomie décisionnelle des parents.

Enfin, une intervenante rapporte une situation où une enfant âgée de six ans est décédée. Celle-ci était alimentée oralement, mais avait d'abondants reflux gastriques, ce qui lui enlevait le goût de manger et diminuait grandement sa qualité de vie. Les recommandations du médecin étaient de mettre en place un gavage, mais les parents ont refusé. Ceux-ci étaient inquiets des implications négatives de cette intervention et étaient réticents en raison de l'espérance de vie limitée de leur fille. Les intervenants, pour leur part, recommandaient cette intervention afin d'améliorer la qualité de vie de la fillette et d'assurer sa survie.

En somme, les refus de traitement de certains parents, lesquels sont relativement rares en clinique, sont des situations susceptibles d'occasionner des dilemmes éthiques chez les intervenantes, notamment lorsque ces refus sont perçus comme pouvant nuire à la santé ou à la sécurité de l'enfant ou même provoquer sa mort.

\section{Suivis partiels des recommandations professionnelles}

D'autres situations cliniques sont rapportées par plusieurs intervenantes comme des sources de dilemmes éthiques. II s'agit des situations où les parents suivent seulement en partie les recommandations professionnelles pour diverses raisons. Encore ici, les intervenantes estiment que ces suivis partiels des recommandations de l'équipe sont susceptibles de mettre en péril la santé, la sécurité ou la vie des enfants concernés. Bien qu'elles valorisent l'autonomie décisionnelle des parents, elles ont parfois de la difficulté à comprendre les motivations des parents qui semblent, considèrent-elles, mettre en danger leur enfant. Les paragraphes qui suivent donnent six exemples de situations cliniques ayant engendré ce genre de dilemmes éthiques.

Par exemple, des intervenantes discutent d'une situation qui concerne une fillette de trois ans alimentée via un tube nasogastrique. Ses parents lui donnaient du lait et d'autres aliments, en dépit des recommandations des intervenants de l'alimenter oralement seulement avec des liquides épaissis et de procéder à une vidéofluoroscopie ${ }^{2}$ pour vérifier s'il y avait des risques d'aspiration ou d'obstruction pour les autres aliments. Pour différentes raisons, les parents de la fillette ont décidé de la dégaver, c'est-à-dire de retirer le tube nasogastrique, et ce, sans obtenir l'aval des intervenants. Dans cette situation, l'intervenante estimait que la collaboration avec les parents pouvait être affectée négativement, car il était difficile pour l'équipe de comprendre les motivations des parents qui semblaient prendre des décisions risquées pour la santé, la sécurité ou même la vie de la fillette.

Une intervenante rapporte une situation vécue avec une enfant âgée de deux ans alimentée par voie orale seulement, dont les parents ne respectaient pas les recommandations de lui donner seulement

\footnotetext{
${ }^{2}$ La vidéofluoroscopie est un examen qui peut être utilisé pour confirmer ou infirmer la présence et la sévérité des anomalies anatomiques ou du processus physiologique altéré lors de la déglutition d'une personne; ce test permet en effet de repérer les anormalités dans la physiologie de la déglutition et d'orienter les interventions [25].
} 
des liquides épaissis et des purées lisses. II a d'ailleurs fallu que la fillette s'étouffe de façon assez importante à la garderie pour que les parents en viennent à respecter les recommandations de l'équipe.

Une participante fait état du cas d'une fillette âgée de deux ans et demi qui était nourrie seulement avec du lait au biberon, car celle-ci avait développé des aversions alimentaires. L'équipe avait mentionné aux parents la nécessité de préparer des purées avec beaucoup de goût pour faire revenir son désir de manger, mais les parents négligeaient de le faire en tout temps. Dans cette situation, l'intervenante estimait que la santé nutritionnelle de l'enfant était en conflit avec le respect de l'autonomie décisionnelle des parents.

Une intervenante rapporte une situation qui concerne un garçon âgé d'un an et demi alimenté exclusivement par voie orale, mais dont les parents ne respectaient pas les recommandations des intervenantes de lui donner uniquement des liquides épaissis. Les intervenantes soupçonnaient la présence de négligence parentale, ce qui complexifiait la situation. Dans cette situation, l'intervenante estimait que la protection des personnes vulnérables était en tension avec le respect de l'autorité parentale.

Une participante mentionne le cas d'un garçon qui avait trois ans et une jeune fille de huit ans. Ces deux enfants étaient alimentés uniquement par voie orale, mais les parents ne respectaient pas les recommandations des intervenantes quant aux textures à leur donner, car ils voulaient que leurs enfants aient une alimentation normale, ce qui favoriserait, estimaient-ils, l'amélioration de leur qualité de vie et leur inclusion sociale. Dans cette situation, l'intervenante considérait que la santé nutritionnelle de l'enfant était en conflit avec la qualité de vie et l'inclusion sociale, voire la notion de normalité.

Enfin, une intervenante fait part d'une situation qui concerne un garçon de six ans gavé par tube nasogastrique depuis sa naissance et dont les parents ne respectaient pas les recommandations de l'équipe. De fait, les parents donnaient des aliments à leur fils par voie orale et refusaient qu'un suivi régulier au plan de l'alimentation soit réalisé par l'équipe. La mère affirmait qu'elle n'avait pas besoin de la présence des intervenants pour alimenter son fils. Dans cette situation, l'intervenante estimait que la santé nutritionnelle de l'enfant était en conflit avec l'autonomie décisionnelle des parents.

Somme toute, ces situations cliniques, bien que rares, sont plus fréquentes que les refus de traitement. Ces cas où des parents suivent partiellement les recommandations professionnelles sont aussi source de dilemmes éthiques chez les intervenantes, car elles estiment que ces parents semblent prendre des décisions qui mettent à risque leur enfant. Les connaissances scientifiques et l'expérience clinique des intervenantes les conduisaient à estimer les risques à la santé, à la sécurité ou à la vie des enfants atteints de dysphagie d'une manière différente de certains parents. Leur degré de tolérance au risque est en général inférieur à celui des parents.

\section{Divergences d'opinions avec des partenaires externes}

Trois intervenantes discutent de dilemmes éthiques liés à des divergences d'opinions avec des partenaires externes. Les paragraphes suivants donnent trois exemples de ce genre de dilemmes éthiques.

Une participante donne l'exemple suivant vécu lors du traitement d'un garçon d'environ neuf mois qui était nourri par gavage. II y avait des différences d'opinions entre l'équipe, qui était du même avis que la mère du garçon, et les partenaires externes. Ces derniers invitaient la mère à donner une certaine quantité quotidienne de lait par gavage. Or, l'enfant régurgitait beaucoup et était constipé lorsqu'il était nourri ainsi. L'équipe était d'accord avec la mère pour qu'elle tente de donner du lait par voie orale et qu'elle puisse compenser ensuite les quantités non prises à la fin de la journée par l'entremise du gavage. Cette différence de points de vue entre des intervenants internes et externes 
au CIUSSS suscitait des dilemmes éthiques, selon cette participante, car l'équipe était partagée entre être fidèle aux recommandations des partenaires externes ou au point de vue de la mère. Autrement dit, l'équipe était déchirée entre l'allégeance ou la loyauté envers les partenaires externes et l'allégeance ou la loyauté envers la mère.

Une intervenante discute d'un garçon âgé de cinq ou six ans qui était gavé. Une vidéofluoroscopie n'avait pas pu être réalisée, mais les partenaires externes avaient tout de même recommandé à l'équipe du CIUSSS de procéder à un entraînement à l'alimentation et à la mère de donner à son enfant des aliments par voie orale. Le garçon a cependant eu des complications au plan respiratoire lorsque la mère lui a donné des liquides, ce qui a conduit les partenaires externes à adhérer par la suite aux avis de l'équipe du CIUSSS. Encore ici, l'équipe était partagée entre être fidèle ou loyale aux recommandations des partenaires externes ou au point de vue de la mère.

Une intervenante rapporte des divergences de points de vue avec le médecin de la région. Elle donne l'exemple d'une fillette de 17 mois pour laquelle un gavage avait été recommandé par l'équipe, en raison du haut risque d'aspirations avec différents types de textures d'aliments. Cette participante mentionne que l'opinion de l'équipe était basée sur la préservation de la sécurité et de la vie de l'enfant, alors que celle du médecin s'appuyait sur le respect de l'autonomie décisionnelle des parents qui ne désiraient pas le gavage.

En bref, les rares divergences d'opinions avec les partenaires externes rapportées par certaines intervenantes peuvent également être source de dilemmes éthiques chez celles-ci.

\section{Stratégies utilisées par les intervenantes}

Les intervenantes font état de deux types de stratégies qu'elles ont utilisées pour résoudre les dilemmes éthiques vécus en clinique, soit : 1) des discussions en équipe; et 2) le recours à de l'aide externe à l'équipe.

\section{Discussions en équipe}

Toutes les intervenantes mentionnent la pertinence et même la nécessité des discussions en équipe pour résoudre les dilemmes éthiques rencontrés. Ces discussions sont utiles pour partager leurs émotions, réduire leur stress ainsi que pour valider ou invalider leurs perceptions des situations et de leurs solutions. De fait, l'appui, la vision et les conseils des membres de l'équipe s'avèrent une aide précieuse dans ces situations de doute éthique. Les intervenantes affirment ne pas soutenir leurs échanges par un modèle de délibération éthique. Certaines parmi elles mentionnent cependant que cela pourrait faciliter la résolution des dilemmes éthiques.

\section{Recours à de l'aide externe à l'équipe}

Plusieurs intervenantes mentionnent avoir eu recours à de l'aide externe à l'équipe pour résoudre les dilemmes éthiques que soulève leur pratique. Par exemple, une participante mentionne consulter au besoin le médecin, le coordonnateur clinique ainsi que divers partenaires externes. Elle indique aussi avoir occasionnellement impliqué un autre parent pour faire état de son expérience, ce qui est souvent pertinent, estime-t-elle, car les parents se sentent ainsi compris par des personnes qui vivent des situations similaires à la leur. Enfin, une autre intervenante mentionne avoir consulté par moments des supérieurs dans des dossiers qu'elle estimait litigieux.

\section{Outils pouvant faciliter la prise de décision éthique}

Toutes les intervenantes rapportent avoir besoin d'aide supplémentaire pour résoudre les dilemmes éthiques qu'elles rencontrent dans le cadre de leur pratique. Comme mentionné plus tôt, bien que rares, ces situations sont vécues difficilement au point où les intervenantes rapportent vivre une certaine détresse. Devant ce constat, plusieurs participantes identifient des outils qui pourraient faciliter, à leur avis, la prise de décisions éthiques dans ces genres de situations et réduire, ce faisant, 
la détresse associée à ces situations. Ces outils sont : 1) obtenir des informations supplémentaires via des examens; 2) discuter avec des intervenants d'autres disciplines; 3) clarifier les rôles et responsabilités des membres de l'équipe; 4) utiliser une méthode pour résoudre les dilemmes éthiques; 5) recourir au service d'une personne formée en éthique; et 6) mettre en place un comité d'éthique clinique au sein de l'établissement.

\section{Obtenir des informations supplémentaires via des examens}

Une participante affirme que l'obtention d'informations supplémentaires par l'entremise d'examens documentant les capacités de l'enfant à s'alimenter et les risques qui sont associés à son alimentation actuelle l'aiderait à résoudre les dilemmes rencontrés. Or, ces examens ne sont pas toujours disponibles pour diverses raisons ou leurs conclusions ne sont parfois pas considérées, en raison de leur absence de validité scientifique dans certaines situations spécifiques où il est difficile de respecter à la lettre le protocole.

\section{Discuter avec des intervenants d'autres disciplines}

Plusieurs intervenantes affirment qu'elles trouveraient pertinent de discuter des dilemmes éthiques qu'elles rencontrent avec des intervenants d'autres disciplines. Par exemple, une participante mentionne que la présence d'un travailleur social pourrait être pertinente à la résolution des dilemmes éthiques. Or, ce professionnel n'est pas toujours impliqué dans les dossiers. Pourtant, sa perspective est souvent riche et utile.

\section{Clarifier les rôles et responsabilités des membres de l'équipe}

Une participante mentionne la pertinence d'offrir une formation à l'équipe sur les rôles et responsabilités des différents professionnels faisant partie de l'équipe, et ce, en lien avec le problème de la dysphagie. Elle mentionne que la clarification de son rôle et de ses responsabilités professionnelles et éthiques au sein de l'équipe serait utile. Notamment, un protocole déterminant plus précisément les situations dans lesquelles elle doit intervenir lui permettrait d'être plus à l'aise dans la résolution des dilemmes éthiques rencontrés.

\section{Utiliser une méthode pour résoudre les dilemmes éthiques}

Une participante mentionne qu'une méthode plus précise pour guider la résolution des dilemmes éthiques serait utile. Une autre intervenante renchérit en affirmant qu'un arbre décisionnel pourrait les aider à résoudre les dilemmes éthiques rencontrés par l'équipe.

\section{Recourir au service d'une personne spécialisée en éthique}

Des participantes sont d'avis que l'accès à une personne spécialisée en éthique serait utile. Par exemple, une participante mentionne que l'accès à une personne ressource ayant une telle expertise pourrait être pertinent pour les soutenir dans la résolution des dilemmes éthiques que soulève leur pratique, car elles s'estiment parfois démunies et peu outillées sur le plan de l'éthique.

\section{Mettre en place un comité d'éthique clinique au sein de l'établissement}

Une participante affirme que l'équipe apprécierait grandement qu'un comité d'éthique clinique soit mis en place au sein du CIUSSS pour les aider à résoudre les dilemmes éthiques rencontrés. Le fait d'avoir accès à une telle ressource serait un atout important, car parfois l'équipe ne sait pas quoi faire et apprécierait un avis externe.

\section{Discussion}

L'objectif de l'étude était de décrire les perceptions d'intervenants des dilemmes éthiques qu'ils rencontrent lors du traitement des problèmes d'alimentation d'enfants souffrant de dysphagie, de même que des manières dont ils les résolvent, le cas échéant. Pour atteindre cet objectif, des données qualitatives ont été recueillies au moyen d'entrevues semi-structurées réalisées auprès de huit intervenantes. Nous estimons que l'objectif de la recherche est partiellement atteint, en ceci que 
la saturation des données n'a pas été obtenue. Comme l'échantillon était constitué de professionnelles de disciplines variées (ergothérapie, orthophonie, nutrition et travail social), il est possible que cette diversité explique, en partie du moins, que la saturation n'ait pas été atteinte.

\section{Dilemmes éthiques inhérents à la pratique}

Bien que la fréquence des dilemmes éthiques soit peu élevée selon les intervenantes, soit en moyenne au moins deux dilemmes par année, les résultats de la recherche révèlent que ceux-ci sont inhérents à la pratique professionnelle et source d'une certaine détresse chez les professionnels. Bushby et ses collaboratrices font le même constat en ergothérapie lorsqu'elles affirment que les dilemmes éthiques ainsi que la détresse éthique sont des tensions inévitables dans le cadre de la pratique ergothérapique [15]. Drolet et Goulet [32] font un constat similaire lorsqu'elles mentionnent que les enjeux éthiques inhérents à la pratique des ergothérapeutes sont source de tensions importantes, notamment de détresse éthique, voire d'épuisement professionnel chez certains ergothérapeutes. Relativement à la faible fréquence des dilemmes éthiques, les résultats de la recherche sont semblables à ceux de Barnitt [33] qui affirme aussi que les situations de refus de traitement sont vécues difficilement par les ergothérapeutes et les physiothérapeutes qu'elle a sondés.

Mentionnons cependant qu'il n'est pas toujours aisé de comparer les résultats de la présente recherche avec ces études étant donné les différences importantes entre leur échantillon. En effet, l'échantillon de la présente recherche était constitué de professionnelles de diverses disciplines préoccupées par les dilemmes éthiques entourant le traitement d'enfants dysphagiques, alors que la méta-analyse de Bushby et ses collaboratrices [15], l'étude de Drolet et Goulet [32] ainsi que celle de Barnitt [33] s'intéressaient aux tensions éthiques suscitées par diverses interventions réalisées auprès de différentes clientèles dans divers milieux en ergothérapie (pour Bushby et ses collaborateurs [15] ainsi que Drolet et Goulet [32]) et en physiothérapie et en ergothérapie (pour Barnitt [33]). Par ailleurs, mentionnons qu'il s'avère souvent complexe de comparer les études entre elles étant donné les grandes diversités méthodologiques qui se présentent entre celles-ci. Cela dit, les paragraphes suivants présentent des comparaisons pouvant être établies entre les résultats de la présente et d'autres réalisées auprès de personnes dysphagiques.

\section{Nature et type de dilemmes éthiques}

Les dilemmes éthiques documentés dans cette étude rejoignent ceux discutés par d'autres intervenants dans les écrits antérieurs en dysphagie. Comme l'observent Craig et Higgs [7], les refus de traitement, les suivis partiels des recommandations professionnelles et les divergences d'opinions entre partenaires sont source de dilemmes éthiques chez les intervenants. Comme l'expliquent ces auteurs, ces situations cliniques résultent du fait que les parents, les intervenants et les partenaires ont parfois des perceptions différentes des risques reliés à l'alimentation orale de l'enfant dysphagique. Par exemple, les parents ont parfois des doutes quant à la validité écologique de certaines évaluations effectuées par les intervenants ou des partenaires, ce qui affecte leur perception du risque. Sur la base de leurs expériences personnelles au quotidien, les parents ont parfois le sentiment d'en connaitre davantage que l'équipe sur la condition dysphagique de leur enfant. De plus, comme le remarquent ces auteurs, c'est comme si les parents étaient détenteurs du risque (risk owners), tandis que les intervenants étaient plutôt gestionnaires du risque (risk managers) [7]. Cette position différente dans la relation avec l'enfant dysphagique et l'expérience limitée avec celui-ci au quotidien peuvent amener certains intervenants à avoir une tolérance au risque inférieure à celle de certains parents. De fait, maints auteurs constatent que les divergences d'opinions sur ces questions entre les intervenants, d'une part, et les parents, d'autre part, sont source de tensions éthiques chez les professionnels [34-36]. Pour faciliter la résolution de ce genre de situations, notamment celles où des divergences d'opinions se présentent, entre l'équipe et les personnes présentant une dysphagie, relativement aux recommandations alimentaires formulées par l'équipe, Kaiser et ses collaborateurs [37] ont développé un algorithme décisionnel afin que l'équipe puisse, en 
collaboration avec les personnes dysphagiques et leur famille, s'entendre sur la diète à privilégier pour assurer une alimentation saine et sécuritaire.

Aussi, comme le remarquent Sharp et Bruant [38], le traitement des personnes dysphagiques est source de questionnements et de dilemmes éthiques chez les intervenants, notamment lors des refus de traitement ou des non-suivis des recommandations professionnelles. Dans ces situations, les intervenants se sentent démunis, questionnent leurs rôles et leurs responsabilités et se demandent dans quelle mesure ces refus et non-suivis sont liés à des consentements véritablement libres et éclairés. C'est pourquoi, dans leur article sur le sujet, Sharp et Bruant [38] expliquent la notion de consentement, différencient les types de consentement (informel ou formel) et proposent une procédure afin d'assurer l'obtention et le respect du consentement libre et éclairé des patients.

Dans le même ordre d'idées, Sharp et Genesen [4] suggèrent un modèle de décision éthique adapté à la gestion des cas cliniques de dysphagie, lequel comprend quatre quadrants qui se présentent comme des lunettes différentes, mais complémentaires à partir desquelles examiner les situations de dilemme éthique. Ces quatre quadrants sont: 1) les indications médicales, 2) les préférences des patients, 3) la qualité de vie de la personne dysphagique et 4) son contexte familial, voire social. Ces auteures estiment qu'au final les préférences des patients devraient avoir préséance en cas de divergences d'opinions entre les intervenants et les parents, notamment parce que le refus de traitement est un droit fondamental et qu'il est important de respecter les préférences des patients.

Huffman et Owre [13] affirment aussi que les refus de traitement, les suivis partiels de recommandations professionnelles et les divergences d'opinions entre intervenants ou partenaires peuvent être source de dilemmes éthiques chez les intervenants. Dans leur article, ils conduisent une analyse éthique de ces situations cliniques et font un survol de différents modèles de délibération éthique pouvant contribuer à la préparation des étudiants à ces réalités cliniques et au soutien des professionnels en exercice qui vivent difficilement ces situations. Ils proposent divers tableaux suggérant des pistes d'action, lesquelles sont en conformité avec les règles déontologiques de l'Association américaine des orthophonistes. Cette façon de procéder, bien que rassurante pour les intervenants, suggère cependant que l'éthique peut se réduire au seul respect de la déontologie professionnelle.

\section{Résolution des dilemmes éthiques}

Les valeurs rapportées par les intervenantes pour justifier leurs décisions et actions sont parfois différentes, quoique similaires, à celles mentionnées dans les études antérieures. Par exemple, dans l'article de Huffman et Owre [13], les intervenants mentionnaient le principe de la bienfaisance, et plus particulièrement, le bien-être et la qualité de vie des patients pour justifier leurs décisions et actions. À l'exception du travailleur social qui accorde en général la primauté à l'autonomie décisionnelle des parents, les valeurs mentionnées par les intervenantes pour justifier leurs décisions et actions sont en général similaires d'une profession à une autre, soit la santé, la sécurité, la protection des personnes vulnérables et la préservation de la vie $[39,40]$.

Toutes les intervenantes mentionnent avoir fait appel aux discussions en équipe pour analyser et résoudre les dilemmes éthiques qu'elles rencontrent dans leur pratique, l'autre stratégie utilisée étant le recours à de l'aide externe à l'équipe (supérieurs, partenaires et parents). La description de ces stratégies utilisées par des intervenants pour surmonter les problèmes éthiques consiste un élément nouveau, en ceci que celles-ci n'ont pas été documentées dans les écrits antérieurs dans les cas de dysphagie à l'enfance, à l'exception du recours aux discussions impliquant plusieurs intervenants $[18,34,41,42]$.

Toutes les intervenantes interviewées s'entendent sur le besoin de moyens supplémentaires pour les aider à résoudre les dilemmes éthiques que pose leur pratique professionnelle. Les moyens supplémentaires suggérés varient toutefois selon les intervenantes, bien que certaines intervenantes 
s'entendent sur certains de ceux-ci. La description des besoins des intervenantes relatifs à l'analyse et à la résolution des dilemmes éthiques est également un aspect nouveau, en ceci que ces besoins du point de vue d'intervenants n'ont pas été spécifiquement décrits dans les écrits antérieurs, à l'exception des besoins relatifs à l'enseignement et à la formation continue $[41,43]$ et à l'utilisation en clinique de modèles ou d'outils pour l'analyse et la résolution des dilemmes éthiques [37,38,41-43]. Ces modèles ou outils ne semblent toutefois pas connus des intervenantes ou, s'ils le sont, ils semblent ne pas combler les besoins des intervenantes interrogées.

\section{Conséquences des dilemmes éthiques}

Toutes les intervenantes rapportent avoir vécu de la détresse devant ces genres de situations. Ce résultat rejoint les travaux de chercheurs qui attestent de la présence somme toute importante de la détresse chez les professionnels de la santé [15,20,32]. Les autres conséquences négatives rapportées dans les écrits n'ont toutefois pas été discutées par les intervenantes [15,17-19,22]. De fait, aucune participante n'a mentionné vivre de l'épuisement professionnel, envisager un changement de carrière, être à la recherche d'un emploi dans un autre établissement ou avoir diminué son nombre d'heures travaillées. II faut dire qu'aucune question ne ciblait ces éléments, ce qui n'est pas le cas de la détresse qui a fait l'objet d'une sous-question spécifique. Cela dit, les résultats attestent de certains aspects des conséquences positives documentées dans les écrits. Notamment, comme l'observe Wiggs [21], les intervenantes font montre d'une conscience de leurs valeurs éthiques. Aussi, comme le constatent McCarthy et Deady [19], les intervenantes envisagent des moyens afin d'améliorer leur façon de résoudre les dilemmes éthiques qu'elles rencontrent. Cependant, sans nullement blâmer leurs supérieurs ni leur établissement, elles ont plutôt tendance à consulter et estimer leurs supérieurs, de même qu'à proposer des outils qui pourraient être mis de l'avant au sein de leur établissement. De fait, leur approche est constructive. Par ailleurs, elles rapportent avoir de bonnes relations entre les membres de leur équipe, plutôt que des discordances.

\section{Forces et limites de la recherche}

Les critères de rigueur scientifique pour une recherche qualitative sont la crédibilité, la transférabilité, la fiabilité et la confirmabilité [26]. Au sujet de la crédibilité, la participation de huit professionnelles de la santé appartenant à quatre professions différentes (ergothérapie, orthophonie, nutrition et travail social) contribue à la crédibilité de la recherche et à la richesse de celle-ci, en fournissant des perspectives différentes et complémentaires de l'objet interrogé, tel que vécu par les participantes. Cela dit, comme pour toute recherche similaire, seules les participantes préoccupées et confrontées à de tels dilemmes ainsi que désireuses de partager leurs perceptions de ceux-ci ont pris part à la recherche. Relativement à la transférabilité, étant donné l'absence de la saturation des données, les conclusions tirées de celle-ci sont difficilement transférables à d'autres personnes et d'autres milieux de pratique. En ce sens, les conclusions reflètent la perspective des participantes, quoiqu'une convergence des points de vue se soit manifestée chez elles. En ce qui a trait à la fiabilité, les entrevues ont été enregistrées sur bande audionumérique et les verbatim transcrits intégralement, ce qui a permis une analyse de contenu collée aux perceptions des participantes, tel qu'en attestent leurs discours. Au sujet de la confirmabilité, les résultats découlant des analyses ont été transmis aux participantes afin qu'ils confirment que ceux-ci reflètent bien leurs points de vue, ce qui contribue à l'exactitude des analyses des données et des interprétations des résultats. Enfin, il aurait été toutefois pertinent de faire usage de multiples sources de données (triangulation) ainsi que d'analyser les verbatim par plusieurs analystes.

\section{Implications de la recherche pour la pratique}

Cette recherche est susceptible d'avoir des implications pour la pratique (clinique, enseignement, recherche et gouvernance). En ce qui a trait à la clinique, la connaissance des principaux dilemmes éthiques entourant le traitement des enfants dysphagiques peut permettre aux intervenants de se préparer à ceux-ci, de les détecter et de s'inspirer ses stratégies et outils documentés ici afin de résoudre de tels dilemmes. Ce faisant, cela peut leur permettre d'être proactifs dans leur approche 
face à ces dilemmes, ce qui a le potentiel de diminuer les conséquences négatives que ceux-ci peuvent engendrer et d'optimiser leurs possibles conséquences positives.

Les résultats de la recherche ont également le potentiel de contribuer à l'enseignement donné, non seulement à des étudiants dans le domaine de la santé (dans le cadre de leurs études universitaires menant à l'exercice de la profession), mais également aux professionnels de la santé œuvrant en dysphagie à l'enfance (dans le cadre de formations continues). En effet, une meilleure connaissance des dilemmes éthiques qui peuvent être rencontrés par les intervenants ainsi que des stratégies et outils pouvant être utilisés par ceux-ci pour résoudre ces dilemmes a le potentiel d'arrimer les enseignements aux réalités cliniques, plutôt que de prêcher dans le vide.

Tel que mentionné précédemment, l'état des connaissances éthiques dans le domaine de l'ergothérapie et des autres disciplines de la réadaptation en matière de dysphagie à l'enfance étant à ce jour limité, les résultats de la recherche contribuent, certes modestement, à l'édification des connaissances éthiques sur ce sujet. Par ailleurs, cette recherche s'inscrit dans le cadre de recommandations d'études antérieures [15,16,32,34], suivant lesquelles des recherches supplémentaires sont requises pour documenter les enjeux éthiques que soulève la pratique professionnelle dans le domaine de la réadaptation. Les résultats ont aussi des implications pour des recherches futures en fournissant des avenues possibles à investiguer. Notamment, la perception des parents et, si possible, des enfants auraient avantage à être documentée, de même que celle d'intervenants d'autres CIUSSS pour confirmer ou non les résultats ici obtenus.

Enfin, les résultats montrent aussi la pertinence que les dirigeants des établissements de santé adressent les problèmes éthiques qui ont lieu en leur sein et soutiennent des solutions visant, notamment (si ce n'est pas déjà fait) à : 1) soutenir les démarches visant à obtenir des informations supplémentaires via des examens; 2) mettre sur pied des tables de rencontres entre les professionnels appartenant à différentes disciplines; 3) clarifier les rôles et les responsabilités de chacun des membres de l'équipe interdisciplinaire; 4) encourager les équipes professionnelles à se munir des outils facilitant la résolution des dilemmes éthiques; 5) soutenir le recours à une personne versée en éthique au besoin; et 6) mettre en place un comité d'éthique clinique au sein de l'établissement. En bref, il nous apparait judicieux que les dirigeants des établissements soient attentifs aux préoccupations éthiques des intervenants et aux solutions que ceux-ci envisagent afin de surmonter les dilemmes éthiques qu'ils vivent dans leur pratique.

\section{Conclusion}

Cette étude a décrit les dilemmes éthiques que soulève le traitement d'enfants présentant des problèmes dysphagiques ainsi que les stratégies et outils pouvant être mis de l'avant afin de résoudre ces dilemmes, et ce, à partir des perceptions d'intervenantes travaillant au sein d'un CIUSSS du Québec. Les situations susceptibles d'occasionner des dilemmes éthiques sont les refus de traitement, les suivis partiels des recommandations professionnelles et les divergences d'opinions avec des partenaires externes. Bien que la fréquence des dilemmes éthiques rapportée par les intervenantes soit somme toute assez faible, toutes les intervenantes sans exception estiment que ces dilemmes leur occasionnent de la détresse. Pour résoudre ces dilemmes éthiques, les intervenantes utilisent principalement les discussions d'équipe, mais ont également recours à de l'aide extérieure à l'équipe (supérieurs, partenaires externes et parents). Aussi, de manière unanime, les intervenantes soulignent le besoin de moyens supplémentaires pour les aider à analyser et à résoudre les dilemmes éthiques qu'ils rencontrent dans le cadre de leur pratique et plusieurs ont formulé des suggestions en ce sens.

Les résultats de la recherche constituent un pas dans l'édification des connaissances par ailleurs limitées relativement à la fréquence des dilemmes éthiques en matière de dysphagie à l'enfance, aux types de dilemmes éthiques que pose cette pratique, aux conséquences négatives que ces dilemmes 
occasionnent ainsi qu'aux stratégies qui sont utilisées, ou qui devraient l'être, par les intervenants pour les résoudre. Cette étude convie d'autres chercheurs à réaliser des recherches supplémentaires dans ce domaine encore peu exploré à ce jour. Plus d'attention devrait être portée à ces dilemmes éthiques en vue de développer des ressources éthiques susceptibles d'aider les intervenants à surmonter ces situations avec aisance et efficacité.

\section{Références}

1. Ordre des ergothérapeutes du Québec (OEQ). Au-delà de la dysphagie, la personne avant tout - Rôle de l'ergothérapeute auprès des personnes présentant des difficultés à s'alimenter ou à être alimentées. Québec: OEQ; 2006.

2. Adverson J, Clark H, Lazarus C, Schooling T, Frymark T. Evidence-based systematic review. Effects of oral motor interventions on feeding and swallowing preterm infants. American Journal of Speech-Language Pathology. 2010;19:321-340.

3. Arcand M, Hébert R. Précis pratique de gériatrie. Québec: Edisem Inc; 2007.

4. Sharp HM, Genesen LB. Ethical decision-making in dysphagia management. American Journal of Speech-Language Pathology. 1996;5(1):15-22.

5. Ordre des ergothérapeutes du Québec (OEQ). Ergothérapie et intervention auprès des personnes présentant des troubles de l'alimentation. Québec: OEQ; 2001.

6. Orthophonie et Audiologie Canada (OAC). Énoncé de position. Le rôle des orthophonistes en dysphagie. 2017.

7. Craig GM, Higgs $P$. Risk owners and risk managers: dealing with the complexity of feeding with neurodevelopmental disability. Health Risk \& Society. 2012;14(7-8):627-637.

8. Arvedson JC, Lefton-Grief MA. Ethical and legal challenges in feeding and swallowing intervention for infants and children. Seminars in speech and language. 2007;28(3):232-238.

9. Swisher LLD, Arslanian LE, Davis CM. The realm-individual process-situation (RIPS) model of ethical decision-making. Hpa resource. Official Publication of the Section on Health Policy \& Administration. 2005;305:284-4535.

10. Tappolet C. Émotions et valeurs. Paris: Presses universitaires de France;2000.

11. Drolet MJ. The axiological ontology of occupational therapy: A philosophical analysis. Scandinavian Journal of Occupational Therapy. 2014;21(1):2-10.

12. Ramé A. La sonde nasogastrique. Revue de l'infirmière. 2007;129:26-27.

13. Huffman NP, Owre DW. Ethical issues in providing services in schools to children with swallowing and feeding disorders. Language, Speech, and Hearing Services in Schools. 2008;39:167-176.

14. Sullivan PB, Juszczak E, Bachlet AME, Thomas AG, Lambert B, Vernon-Roberts A, Grant HW, Eltumi M, Alder N, Jenkinson C. Gastrostomy feeding in cerebral palsy: too much of a good thing? Developmental Medicine \& Child Neurology. 2004;46:796-800.

15. Bushby K, Chan J, Druif S, Ho K, Kinsella EA. Ethical tensions in occupational therapy practice: a scoping review. British Journal of Occupational Therapy. 2015;78(4):212-221.

16. Kinsella EA, Park A, Appiagyei J, Chang E, Chow D. Through the eyes of students: ethical tensions in occupational therapy practice. Canadian Journal of Occupational therapy. 2008;75(3):176-183.

17. Radzvin LC. Moral distress in certified registered nurses anesthesists: implications for nursing practice. American Association of Nurse Anesthesists Journal. 2011;79(1):39-45.

18. Lefton-Grief MA, Arvedson JC. Ethical considerations in pediatric dysphagia. Seminars in speech and language. 1997;18(1):79-87.

19. McCarthy J, Deady R. Moral distress reconsidered. Nursing Ethics. 2008;15(2):254-262.

20. Kälvemark S, Höglund AT, Hansson MG, Westerholm P, Arnetz B. Living with conflicts-ethical dilemmas and moral distress in the health care system. Social Science \& Medicine. 2004;58: 1075-1084.

21. Wiggs CM. Case study Baby John - nursing reflections on moral angst. Nursing Ethics. 2011;18(4):606-612. 
22. Edwards H, Dirette D. The relationship between professional identity and burnout among occupational therapists. Occupational Therapy in Health Care. 2010;24:119-29.

23. Kaiser F, Spiridigliozzi AM, Hunt MR. Promoting shared decision-making in rehabilitation: development of a framework for situations when patients with Dysphagia refuse diet modification recommended by the treating team. Dysphagia. 2012;27(1):81-87.

24. DePoy E, Giltin, LN. Introduction to Research: Understanding and Applying Multiple Strategies. $5^{e}$ éd. Missouri: Elsevier; 2010.

25. Husserl E. The Crisis of European Sciences and Transcendental Phenomenology. Illinois: Northwestern University Press; 1970.

26. Fortin MF. Fondements et étapes du processus de recherche. Montréal: Chenelière Éducation; 2010.

27. Corbière $M$, Larivière $N$. Méthodes qualitatives, quantitatives et mixtes dans la recherche en sciences humaines, sociales et de la santé. Québec: Presses de l'Université du Québec; 2014.

28. Thomas SP, Pollio HR. Listening to Patients. A Phenomenological Approach to Nursing Research and Practice. New York: Springer Publishing Company; 2002.

29. Giorgi A. The theory, practice and evaluation of the phenomenological method as a qualitative research procedure. Journal of Phenomenological Psychology. 1997;28(2):235-260.

30. Peladeau-Pigeon M, Steele CM. Aspects techniques de l'étude vidéofluoroscopique de la déglutition. Revue canadienne d'orthophonie et d'audiologie. 2013;37(3):216-226.

31. Flori N, Gilles V, Bouteloup C, Senesse P. La gastrostomie : quelle technique pour tel patient? Nutrition clinique et métabolisme. 2011;25(1):36-40.

32. Drolet MJ, Goulet M. Les barrières et facilitateurs à l'actualisation des valeurs professionnelles : perceptions d'ergothérapeutes du Québec. Recueil annuel belge francophone d'ergothérapie. 2017;9:7-42.

33. Barnitt R. Ethical dilemmas in occupational therapy and physical therapy: a survey of practitioners in the UK National Health Service. Journal of Medical Ethics. 1998;24:193-199.

34. Craig GM, Scambler G, Spitz L. Why parents of children with neurodevelopmental disabilities requiring gastrostomy feeding need more support. Development Medicine \& Child Neurology. 2003;45:183-188.

35. Miller-Thiel J, Glover JJ, Beliveau E. Caring for the dying child. The Hospice Journal. 1993;9(2-3):55-73.

36. Townsley R, Robinson C. More than just a health issue: a review of current issues in the care of enterally-fed children living in the community. Health and Social Care, 1999;7(3):216-224.

37. Kaiser F, Spiridigliozzi AM, Hunt MR. Promoting shared decision-making in rehabilitation: development of a framework for situations when patients with dysphagia refuse diet modification recommended by the treating team. Dyspharia, 2012;27:81-87.

38. Sharp HM, Bruant KN. Ethical issues in dysphagia: When patients refuse assessment or treatment. Seminars in Speech and Language. 2003;24(4):285-299.

39. Université Laval. Les professions de la relation d'aide : comment les différencier? Direction des services aux étudiants, 2012.

40. Conseil de recherches en sciences humaines du Canada, Conseil de recherches en sciences naturelles et en génie du Canada, Instituts de recherche en santé du Canada. Énoncé de politique des trois conseils. Éthique de la recherche avec des êtres humains, décembre 2014.

41. Hudon A, Laliberté M, Hunt M, Saunier B, Wlliams-Jones B, Mazer B. What place for ethics? An overview of ethics teaching in occupational therapy and physiotherapy programs in Canada. Journal of Disability and Rehabilitation. 2014;36(9):775-80.

42. Chabon SS, Morris JF. A consensus model for making ethical decisions in a less-than-ideal world. The ASHA Leader. 2004;9(3):18-19.

43. Drolet M-J, Hudon A. Theoretical frameworks used to discuss ethical issues in private physiotherapy practice and proposal of a new ethical tool. Medicine, Health Care and Philosophy. 2015;18(1):51-62. 